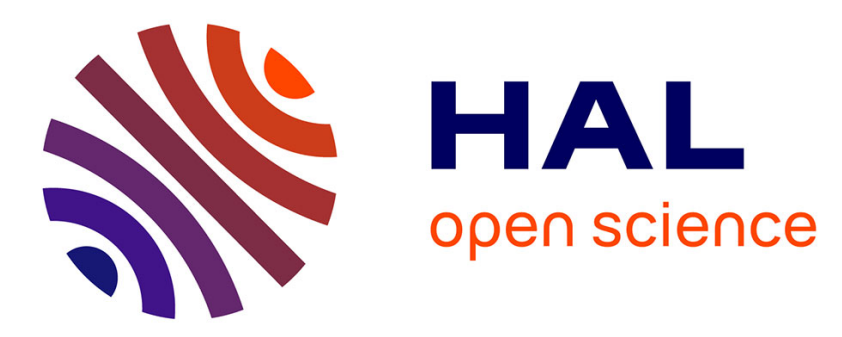

\title{
Pulse doubling and wavelength conversion through triangular nonlinear pulse reshaping
}

\author{
Noémie Verscheure, Christophe Finot
}

\section{To cite this version:}

Noémie Verscheure, Christophe Finot. Pulse doubling and wavelength conversion through triangular nonlinear pulse reshaping. Electronics Letters, 2011, 47 (21), pp.1194-1196. 10.1049/el.2011.2527 . hal-00626183

\section{HAL Id: hal-00626183 \\ https://hal.science/hal-00626183}

Submitted on 23 Sep 2011

HAL is a multi-disciplinary open access archive for the deposit and dissemination of scientific research documents, whether they are published or not. The documents may come from teaching and research institutions in France or abroad, or from public or private research centers.
L'archive ouverte pluridisciplinaire HAL, est destinée au dépôt et à la diffusion de documents scientifiques de niveau recherche, publiés ou non, émanant des établissements d'enseignement et de recherche français ou étrangers, des laboratoires publics ou privés. 


\title{
Pulse doubling and wavelength conversion through triangular nonlinear pulse reshaping
}

\author{
N. Verscheure and C. Finot
}

We present a proof of principle experiment demonstrating the benefits of using a triangular temporal profile in the context of copying and wavelength conversion of telecommunication signals. Generated by passive nonlinear reshaping in a set of two carefully chosen fibres, the triangular shape enables efficient temporal and spectral doubling of the signals through self-phase modulation.

Introduction: All-optical wavelength conversion is a key function for the development of future transparent networks. Among the various photonic solutions that have been proposed so far, Kerr nonlinearity in highly nonlinear fibres seems particularly attractive. Several approaches using four-wave mixing or self- / cross- phase modulation (SPM / XPM) followed by offset spectral filtering have been reported [1, 2]. SPM and XPM are highly affected by the temporal intensity profile of the pulse and, consequently, the wavelength conversion performance will depend on the initial pulse shape. In particular, temporal triangular or sawtooth (asymmetric triangular) profiles are very promising candidates for achieving clean and efficient conversion [1, 2].

The problem of generating such non-conventional pulse shapes has attracted a good deal of attention in recent years. Various solutions have been proposed, including linear shaping methods (superstructured fibre Bragg gratings [1, 2] or frequency-totime conversion [3]) and nonlinear pulse shaping in photonic crystal fibre [4] or normally dispersive (ND) fibre [5]. 
In [5], Boscolo et al have numerically shown that an initial, sufficiently powerful pulse with anomalous linear chirp can evolve towards a triangular shape in a passive, ND fibre. In contrast with parabolic pulses that can be generated through the asymptotic pulse reshaping occurring upon nonlinear propagation in ND fibre amplifiers [6], triangular pulses represent transient states of the nonlinear pulse evolution in a passive ND fibre. Additional experimental studies have confirmed that a precise trade-off between the system parameters (initial chirp value, peak power, fibre length) is required for triangular pulse formation [7].

In this Letter, we experimentally demonstrate spectral and temporal optical pulse doubling and frequency conversion using triangular pulses generated in ND fibre. We show that the frequency/time spacing between the two pulse replica can be easily tuned by control of the initial power.

Experimental set-up: The all-fibred experimental set-up relies exclusively on telecommunication-ready devices and is sketched in Fig. 1, which also includes the parameters of the fibre segments being used. A 2.4 ps soliton pulse train is delivered by a mode-locked fibre laser (MLFL) operating at a repetition rate of $10 \mathrm{GHz}$ (see inset of Fig. 1). An intensity modulator (IM) is used to decrease the repetition rate down to $2.5 \mathrm{GHz}$. The reshaping of the initial sech pulses into triangular pulses is achieved through propagation in a set of two carefully chosen fibres [7]. The choice of the fibres was guided by the design rules proposed in [5], which were slightly modified to account for the non-Gaussian shape of the initial pulses. The first stage of propagation is to impose an anomalous chirp on the pulses and relies on linear propagation in a standard single mode fibre (SMF). The pre-chirped, broadened pulses are amplified by an erbium-doped fibre amplifier (EDFA) with an output average power of $20 \mathrm{dBm}$, and then propagated through a non-zero dispersion shifted fibre (NZ-DSF) with normal dispersion to realize the nonlinear triangular pulse 
reshaping. The output triangular pulses from the NZ-DSF are amplified by a highpower EDFA before entering a highly nonlinear fibre (HNLF). A fibre with normal dispersion was favoured to avoid the modulational instability effects that can occur in the anomalous dispersion regime. This stage of propagation is dominated by SPM and the nonlinear phase shift generated by an intensity profile with linear gradient [1] : the linear leading (trailing) edge of the triangular pulse induces a constant, red (blue) frequency shift. The output SPM-shaped spectrum from the HNLF is sent into either a linear SMF segment to realize temporal pulse doubling or a wavelength demultiplexer filter for wavelength conversion processing. The filter channels have a full-width at half-maximum (FWHM) of $100 \mathrm{GHz}$ and are spaced by $200 \mathrm{GHz}$.

At each propagation stage, the temporal intensity profiles of the pulses are monitored by an optical sampling oscilloscope (OSO) with picosecond temporal resolution. The optical spectra are also recorded.

Temporal and spectral evolutions: The temporal and spectral pulse evolutions are summarized in Fig. 2. After propagation in the SMF, the initial transform-limited pulses are broadened up to $7.1 \mathrm{ps}$ (Fig. 2a). The unchanged spectrum at the SMF output confirms the purely linear nature of the propagation in this fibre segment. At the output of the NZ-DSF, the pulses clearly exhibit the expected reshaping with a typical triangular intensity profile with a FWHM duration of 34 ps (Fig. 2b). Although deviations from a perfect triangular shape are noticeable on the pulse top, the highly linear wings of the profile qualitatively confirm the conclusions drawn from frequency resolved optical gating measurements in [7].

Due to the triangular temporal profile along with SPM, upon propagation in the HNLF the pulse spectrum goes through a reshaping leading to a structure consisting of two equal peaks. Results are presented in Fig. 2c for an average power at the HNLF input of $24.4 \mathrm{~dB}$. The two spectral peaks at the end of the HNLF contain most of the 
pulse energy and are separated by $600 \mathrm{GHz}$. It is also seen from Fig. 2c that the temporal triangular profile is maintained over propagation in the HNLF.

Spectral and temporal signal copying and wavelength conversion: Additional measurements were made to highlight the influence of the optical power launched into the HNLF on the spectral reshaping process. The evolution of the output spectrum from the HNLF as a function of the launched average power is presented in Fig. 3a. It can be clearly seen that for powers ranging from $18.5 \mathrm{dBm}$ to $28.5 \mathrm{dBm}$, the spectrum exhibits the typical signature of SPM-induced reshaping of a triangular pulse, i.e. a double peak structure. Note that in Ref. [1] sawtooth pulses were voluntarily used to promote the transfer of energy to a single spectral peak. The spectral structure that we observe here is nearly symmetric with respect to the input pulse wavelength. The width and form of the two spectral peaks is almost preserved over the aforementioned range of initial powers and quite remarkably, the separation between the peaks can be tuned continuously between $300 \mathrm{GHz}$ and $900 \mathrm{GHz}$ [8]. The nearly linear variation of the frequency spacing with the initial power is illustrated in Fig. 3b.

By use of linear propagation in the SMF segment following the HNLF, we were able to realize the function of temporal pulse copying as theoretically predicted in [8]. Under propagation of the modulated output pulse from the HNLF in the SMF, the produced spectral separation of the pulse components leads to their subsequent separation in time, thus allowing temporal replication of the pulse. The evolution of the temporal intensity profile recorded at the output of the SMF with the power launched into the HNLF is presented in Fig. $3 \mathrm{c}$ and shows that, as a result of the time-to-frequency mapping occurring in the SMF, the time separation between the two copies of the original pulse can be adjusted by control of the input power to the HNLF. 
We have also investigated the quality of the pulse trains obtained from spectral filtering of the output pulses from the HNLF. Converted pulses trains obtained for the frequency spacings $400 \mathrm{GHz}(1548.51 \mathrm{~nm}$ and $1545.32 \mathrm{~nm}$, respective ITU channels 36 and 40 on a $100 \mathrm{GHz}$ grid spacing) and $800 \mathrm{GHz}$ (ITU channels 34 and 42) have been measured and no significant amplitude or timing jitters have been detected. The output FWHM pulse duration measured on the OSO was $9.7 \mathrm{ps}$. The electrical eyes corresponding to a 10 Gbps pulse train formed by a pseudo random bit sequence with $25 \%$ of 1 bits are presented in Fig. $3 d$ for the ITU channels 34 and 42 . Further bit error rate (BER) measurements have confirmed that error-free operation of the wavelength converter can be achieved.

Conclusion: We have experimentally demonstrated an easy-to-implement all-fibre wavelength converter using triangular-shaped pulses. The nonlinear approach presented takes advantage of the SPM experienced by triangular pulses in a HNLF and enables efficient splitting of the optical spectrum into two symmetric, equal peaks whose spacing can be easily controlled through the input power. The quality of the obtained signals has been assessed by BER measurements. The principle of temporal pulse doubling has also been experimentally validated.

While the proof-of-principle experiments presented in this paper rely on optical fibres, we can anticipate that the rapid recent progress in highly nonlinear waveguide fabrication may enable integration of such high-speed wavelength conversion function based on Kerr nonlinearity on a photonic chip. 
Acknowledgements: This research was supported by the Conseil Regional de Bourgogne (PARI PHOTCOM). We thank Sonia Boscolo and Julien Fatome for stimulating discussions as well as Philippe Morin for technical help in the BER measurements. We also acknowledge the support of the Ministère des affaires étrangères (Alliance Grant 22836ZJ). 


\section{References}

1. Parmigiani F, Ibsen M, Ng TT, Provost L, Petropoulos P, Richardson DJ: 'An efficient wavelength converter exploiting a grating based saw-tooth pulse shaper', IEEE Photon Technol Lett, 2008, 20, pp 1461-1463.

2. Parmigiani F, Ibsen M, Petropoulos P, Richardson DJ: 'Efficient all-optical wavelength conversion scheme based on a saw-tooth pulse shaper', IEEE Photon Technol Lett, 2009, 21, pp. 1837-1839.

3. Ye J, Yan L, Pan W, Luo B, Zou X, Yi A, et al.: 'Photonic generation of triangular-shaped pulses bases on frequency to time conversion', Opt Lett, 2011, 36, pp. 1458-1460.

4. Bale BG, Boscolo S, Hammani K, Finot C: 'Effects of fourth-order fiber dispersion on ultrashort parabolic optical pulses in the normal dispersion regime', $J$ Opt Soc Am B, 2011, 28, pp. 2059-2065.

5. Boscolo S, Latkin Al, Turitsyn SK: 'Passive nonlinear pulse shaping in normally dispersive fiber systems', IEEE J Quantum Electron, 2008, 44, pp. 11961203.

6. Finot C, Dudley JM, Kibler B, Richardson DJ, Millot G: 'Optical parabolic pulse generation and applications', IEEE J Quantum Electron, 2009, 45, pp. 1482-1489.

7. Wang $\mathrm{H}$, Latkin $\mathrm{Al}$, Boscolo S, Harper P, Turitsyn SK: 'Generation of triangular-shaped optical pulses in normally dispersive fibre', $J O p t, 2010,12$, pp. 035205.

8. Latkin A, Boscolo S, Bhamber RS, Turitsyn SK: 'Doubling of optical signals using triangular pulses', J Opt Soc Am B, 2009, 26, pp. 1492-1496. 


\section{Authors' affiliations:}

Noémie VERSCHEURE and Christophe FINOT * (Laboratoire

Interdisciplinaire Carnot de Bourgogne, UMR 5209 CNRS-Université de Bourgogne, 9 Av. A. Savary, BP 47 870, F-21078 DIJON Cedex, FRANCE)

* e-mail adress of the corresponding author : christophe.finot@ u-bourgogne.fr 


\section{Figure captions:}

Fig. 1 Experimental set-up and parameters of the fibre segments (second-order dispersion $\mathrm{D}$, nonlinear coefficient $\gamma$ and length $\mathrm{L}$ ). Inset : Temporal intensity profile of the initial pulses delivered by the fibre laser.

Fig. 2 Snapshots of the temporal (subplots 1) and spectral (subplots 2) intensity profiles at different stages of propagation : (a) after the SMF, (b) after the NZ-DSF and (c) after the HNLF. The profiles are normalized to the maximum intensity.

Fig. 3 (a) Evolution of the spectral intensity profile at the output of the HNLF with the average input power. (b) Dependence of the frequency spacing between the two peaks of the output spectrum from the HNLF on the input power. (c) Evolution of the temporal intensity profile recorded after the second SMF segment with the average input power to the HNLF. (d) Eye diagrams of the PRBS pulse train obtained after spectral filtering for $800 \mathrm{GHz}$ spacing (ITU channels 34 and 42 on subplots $\mathrm{d} 1$ and d2, respectively). 
Figure 1

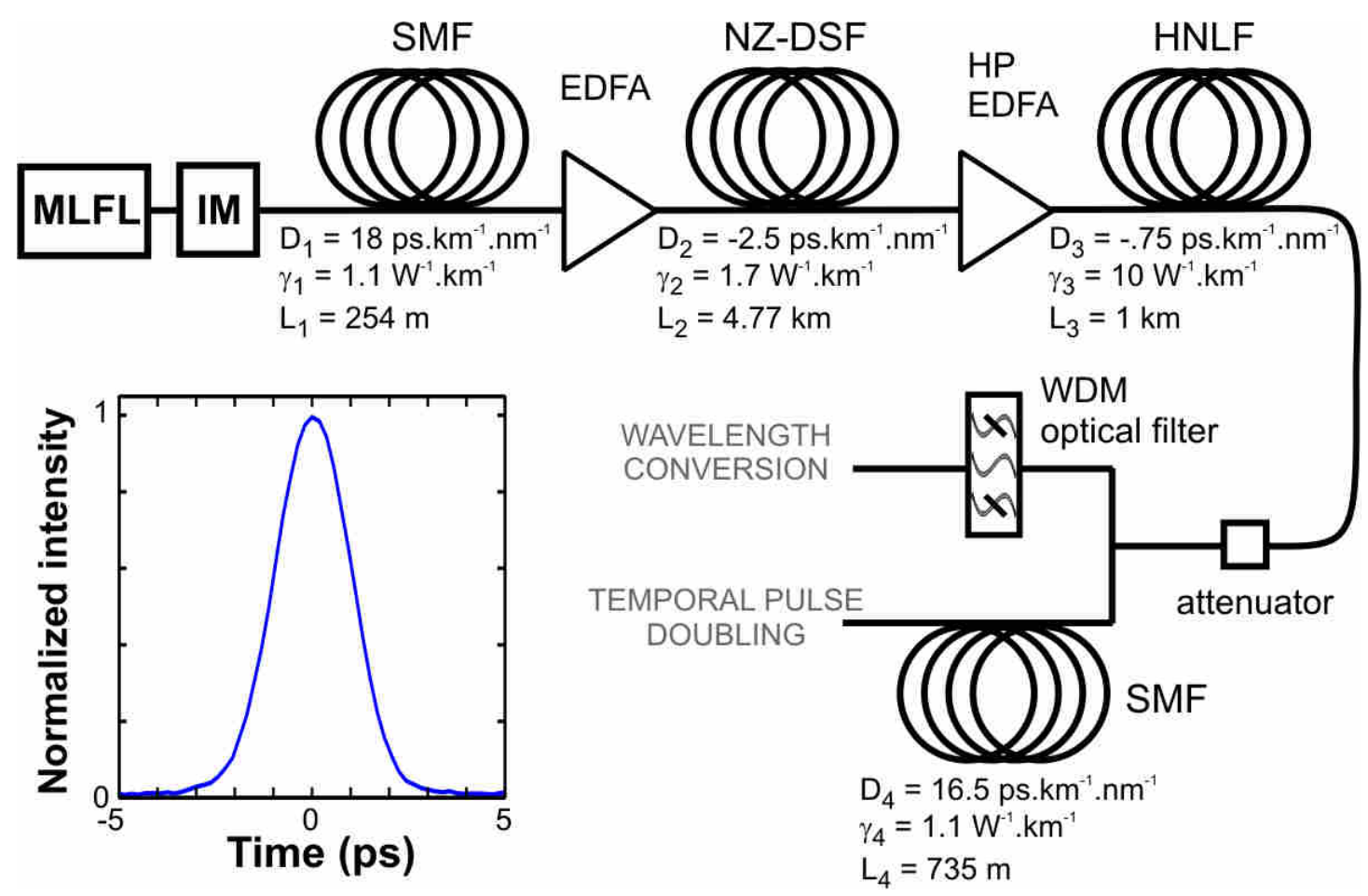


Figure 2
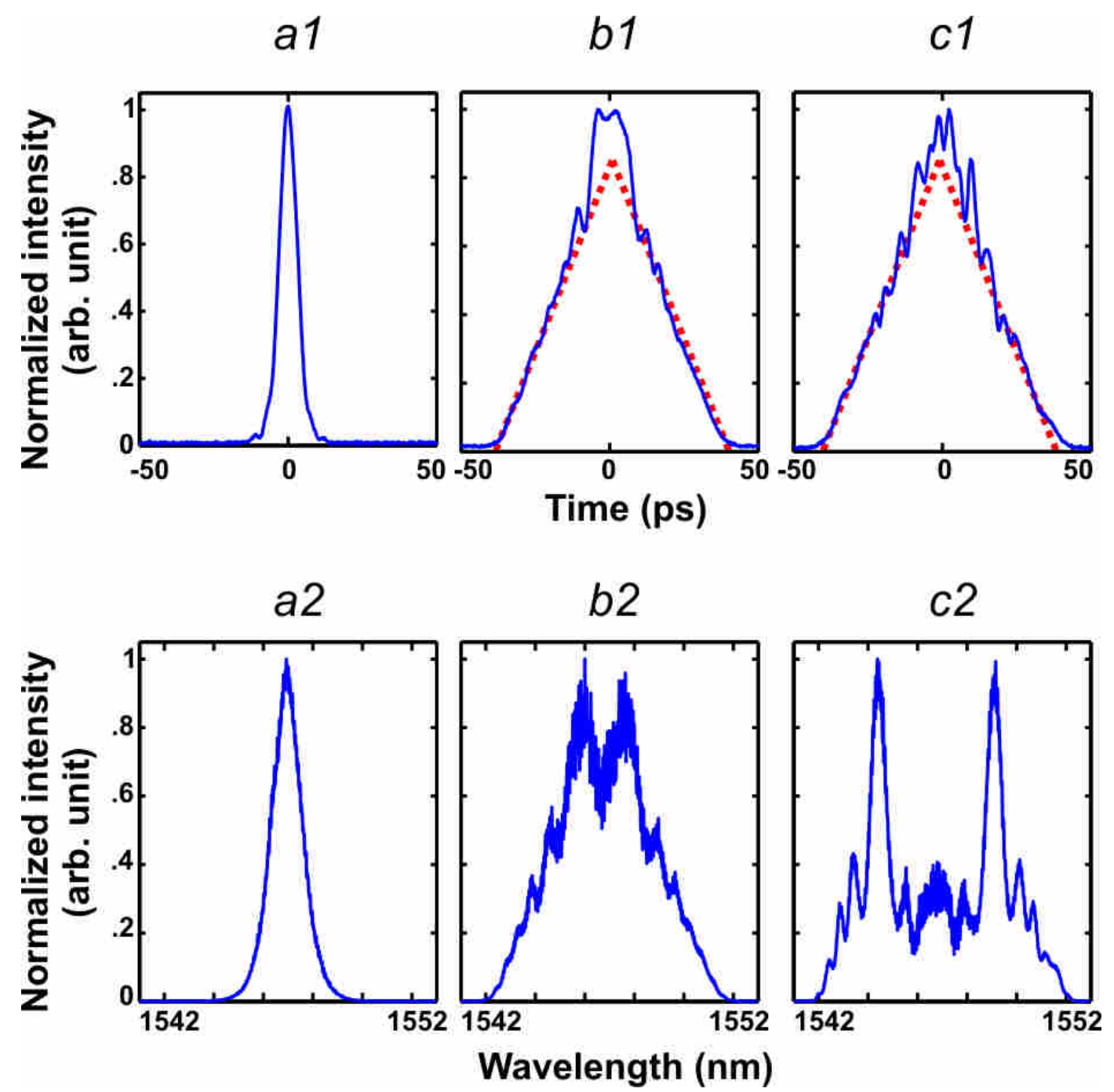
Figure 3

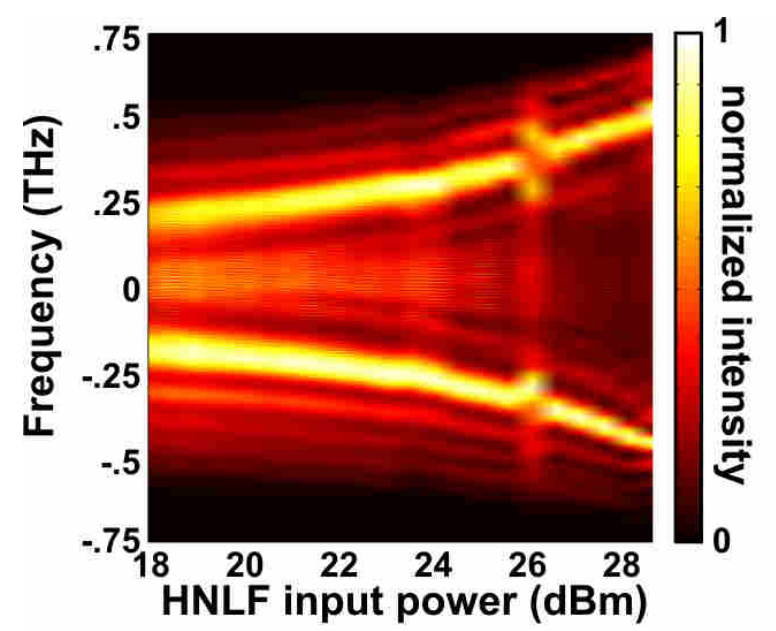

a

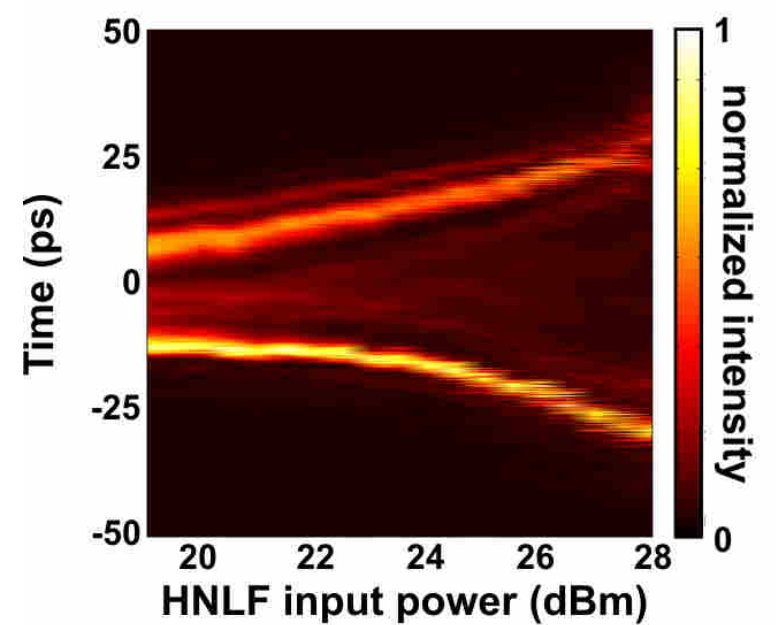

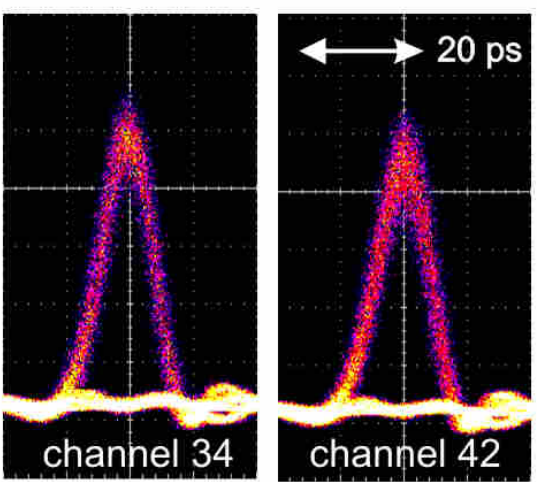

d1

$d 2$

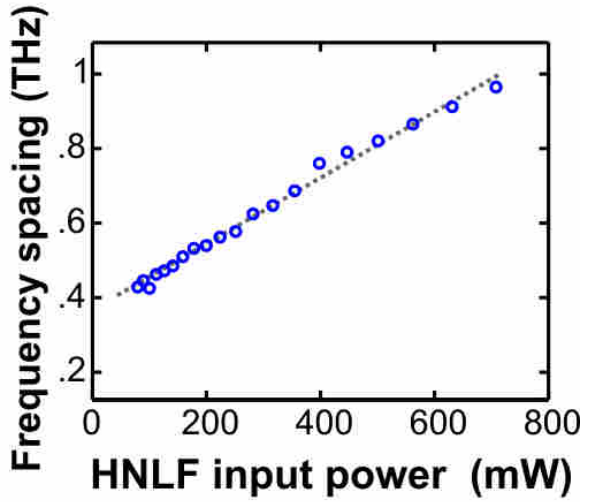

b

channel 42 